\title{
Pediatric Atypical Teratoid/Rhabdoid Tumors of the Brain: Identification of Metabolic Subgroups Using In Vivo 'H-MR Spectroscopy
}

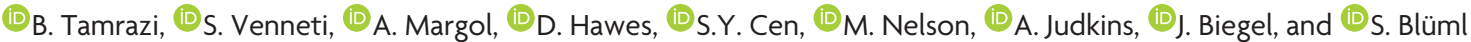

\begin{abstract}
BACKGROUND AND PURPOSE: Atypical teratoid/rhabdoid tumors are rare, aggressive central nervous system tumors that are predominantly encountered in very young children. Our aim was to determine whether in vivo metabolic profiles correlate with molecular features of central nervous system pediatric atypical teratoid/rhabdoid tumors.
\end{abstract}

MATERIALS AND METHODS: Twenty confirmed patients with atypical teratoid/rhabdoid tumors who underwent MR spectroscopy were included in this study. In vivo metabolite levels of atypical teratoid/rhabdoid tumors were compared with molecular subtypes assessed by achaete-scute homolog 1 expression. Additionally, brain-specific creatine kinase levels were determined in tissue samples.

RESULTS: In vivo creatine concentrations were higher in tumors that demonstrated achaete-scute homolog 1 expression compared with those without achaete-scute homolog 1 expression ( $3.42 \pm 1.1$ versus $1.8 \pm 0.8 \mathrm{IU}, P<.01)$. Additionally, levels of myo-inositol (ml) ( $9.0 \pm$ 1.5 versus $4.7 \pm 3.6 \mathrm{IU}, P<.05$ ) were significantly different, whereas lipids approached significance ( $44 \pm 20$ versus $80 \pm 30 \mathrm{IU}, P=.07$ ) in these 2 cohorts. Higher brain-specific creatine kinase levels were observed in the cohort with achaete-scute homolog 1 expression $(P<$ .05). Pearson correlation analysis showed a significant positive correlation of brain-specific creatine kinase with absolute creatine $(P<.05)$ and myo-inositol $(P<.05)$ concentrations.

CONCLUSIONS: In vivo MR spectroscopy may predict key molecular features of atypical teratoid/rhabdoid tumors at initial diagnosis, leading to timely patient risk stratification and accelerating the development of targeted therapies.

ABBREVIATIONS: ASCLI = achaete-scute homolog 1; AT/RT = atypical teratoid/rhabdoid tumor; $\mathrm{CKB}=$ brain-specific creatine kinase; $\mathrm{Cr}=\mathrm{creatine} ; \mathrm{ml}=$ myo-inositol; Cho = choline; SMARCB1 = SWI/SNF-related matrix-associated actin-dependent regulator of chromatin subfamily B member 1

$\mathbf{P}$ rimary brain tumors are the most common pediatric solid tumors in children and the leading cause of death from childhood cancer. ${ }^{1,2}$ Recently, through the emergence of molecular diagnostics and the growing field of pediatric molecular neurooncology, biologically distinct and clinically relevant molecular subtypes of pediatric brain tumors have been identified. These include atypical teratoid/rhabdoid tumors (AT/RTs), a group of rare, highly aggressive central nervous system tumors with poor

Received December 13, 2018; accepted February 27, 2019

From the Departments of Radiology (B.T., M.N., S.B.) and Pediatrics (A.M.) and Division of Hematology Oncology, and Pathology (D.H., A.J., J.B.), Children's Hospital Los Angeles, Los Angeles, California; Department of Pathology (S.V.), University of Michigan, Ann Arbor, Michigan; Department of Radiology and Neurology (S.Y.C.), University of Southern California, Los Angeles, California; and Rudi Schulte Research Institute (S.B.), Santa Barbara, California.

Funding for this research provided by the American Society of Pediatric Neuroradiology Research Award.

Please address correspondence to Benita Tamrazi, MD, Children's Hospital Los Angeles, Department of Radiology, 4650 Sunset Blvd, Los Angeles CA 90027 MS 81; e-mail: btamrazi@chla.usc.edu

http://dx.doi.org/10.3174/ajnr.A6024 prognosis. The genetic hallmark of AT/RTs is the loss of protein expression of SWI/SNF-related matrix-associated actin-dependent regulator of chromatin subfamily B member 1 (SMARCB1), a tumor-suppressor locus on chromosome 22q11.23. ${ }^{3,4}$ Although these tumors are uniformly characterized by deletions and/or mutations in this locus, there is clinical and molecular heterogeneity among the patients. This suggests further underlying biologic heterogeneity, ${ }^{5,6}$ which has been defined by recent multicenter studies. ${ }^{7,8}$ Noninvasive imaging markers obtained at initial presentation can potentially serve as surrogates for these molecular subgroups or for specific clinically relevant molecular features that may lead to patient risk stratification and improved treatment strategies. Recently, an imaging study demonstrated significant differences in the location and conventional MR imaging characteristics of AT/RTs, which may potentially correlate with the different molecular features/subgroups. ${ }^{9}$

Among the molecular features that can be used to distinguish AT/RTs is a transcription factor known as achaete-scute homolog 1 (ASCL1). Patients whose tumors had expression of ASCL1 had improved overall survival. ${ }^{10}$ In this study, we focused on this spe- 
Table 1: Patient demographics and clinical and biologic lesion characterization

\begin{tabular}{|c|c|c|c|c|c|c|c|}
\hline Age (yr) & Sex & Overall Survival (mo) & Location & LM & ASCL1 & CKB & Germline Mutation \\
\hline 0.15 & $\mathrm{~F}$ & $0.5^{\mathrm{a}}$ & Posterior fossa & 0 & Negative & 5.05 & \\
\hline 0.32 & $\mathrm{~F}$ & 17.0 & Posterior fossa & 0 & Positive & 6.31 & Yes \\
\hline 0.39 & $M$ & $8.2^{\mathrm{b}}$ & Posterior fossa & 1 & Positive & & \\
\hline 0.64 & M & 7.0 & Parietal lobe & 0 & Negative & 5.10 & \\
\hline 0.67 & M & $0.5^{\mathrm{a}}$ & Posterior fossa & 1 & Positive & & \\
\hline 0.68 & M & 5.1 & Intraventricular & 0 & ND & & \\
\hline 0.92 & $\mathrm{~F}$ & $0.5^{\mathrm{a}}$ & Posterior fossa & 1 & Positive & & Yes \\
\hline 0.95 & M & $71.9^{b}$ & Posterior fossa & 0 & ND & & \\
\hline 1.09 & M & 13.0 & Posterior fossa & 0 & Negative & 5.31 & \\
\hline 1.14 & M & 7.0 & Temporal lobe & 0 & Positive & 6.05 & Yes \\
\hline 1.15 & M & 59.0 & Posterior fossa & 0 & Negative & 6.31 & \\
\hline 1.42 & $\mathrm{~F}$ & 9.0 & Posterior fossa & 1 & ND & & \\
\hline 1.54 & M & 32.0 & Temporal lobe & 0 & Positive & 6.53 & Yes \\
\hline 1.55 & M & $63.2^{\mathrm{b}}$ & Posterior fossa & 0 & Negative & & \\
\hline 1.73 & $M$ & 13.0 & Pineal & 0 & Positive & & \\
\hline 1.84 & $\mathrm{~F}$ & 18.0 & Frontotemporal & 0 & Negative & 5.21 & \\
\hline 1.96 & $\mathrm{~F}$ & NA & Temporal lobe & 1 & ND & & \\
\hline 2.86 & $M$ & 18.0 & Frontotemporal & 1 & Positive & 6.61 & \\
\hline 7.43 & $\mathrm{~F}$ & 40.0 & Frontotemporal & 0 & Positive & 6.42 & \\
\hline 13.72 & M & 40.0 & Intraventricular & 0 & Positive & 5.71 & \\
\hline
\end{tabular}

Note:-NA indicates patient lost to follow up; LM, leptomeningeal disease present (1) versus absent (0) at presentation; ND, not determined.

a Patients who died from surgical/other complications with no MR imaging or clinical evidence of progressive tumor.

b Patients who were still alive at the time of the completion of this study.

cific molecular feature and explored whether in vivo ${ }^{1} \mathrm{H}-\mathrm{MR}$ spectroscopy can distinguish ASCL1-expressing AT/RTs from those that do not express ASCL1. In an analysis of tissue samples, we observed different levels of brain-specific creatine kinase (CKB), with CKB being higher in ASCL1-expressing AT/RTs. Thus, our primary objective was to determine whether in vivo $\mathrm{Cr}$ concentrations were significantly higher in AT/RTs with ASCL1 expression versus tumors without ASCL1 expression. A secondary goal of this study was to review other metabolic features that are obtained simultaneously with the measurement of creatine to further differentiate these 2 cohorts using a metabolic/MR spectroscopy imaging approach.

\section{MATERIALS AND METHODS \\ Patients}

Our single-center, retrospective study was approved by our institutional review board (Children's Hospital Los Angeles) and was compliant with the Health Insurance Portability and Accountability Act. The requirement to obtain informed consent was waived. We accessed the pediatric oncology data base of our institution to identify patients with AT/RTs from 2000 to 2017 with MR spectroscopy and frozen tissue specimens obtained at our institution. All included patients had confirmed AT/RTs of the brain with patient characteristics summarized in Table 1.

\section{Imaging Protocol}

All brain MR spectroscopy examinations were performed with 1.5T or 3T whole-body MR imaging systems in our institution (Achieva and Ingenia, Philips Healthcare, Best, the Netherlands; Signa HD 1.5, GE Healthcare, Milwaukee, Wisconsin). A singlevoxel point-resolved spectroscopic sequence with $\mathrm{TE}=35 \mathrm{~ms}$, $\mathrm{TR}=1.5$ seconds (for $1.5 \mathrm{~T}$ ) and 2 seconds (for $3 \mathrm{~T}$ ), and 128 signal averages was used for all acquisitions. T2-weighted fast spin-echo, FLAIR, and T1-weighted FLAIR images were acquired in all instances before MR spectroscopy and were reviewed to determine the extent of tumor lesions. We identified the ROIs for MR spectroscopy on the basis of MR images from all 3 planes centered in the solid parts of the tumors, excluding any surrounding normal brain tissue or edema and in a manner that minimized inclusion of cystic or necrotic areas. An attending pediatric neuroradiologist reviewed and approved the ROIs for each patient and confirmed that the MR spectroscopy profile was representative of tumor tissue only. The sizes and shapes of the ROIs were adjusted to lesion size and typically varied between 5 and $10 \mathrm{~cm}^{3}$. Spectra were processed using the fully automated LCModel, Version 6.1-1 L software (http://www.lcmodel.com/). Absolute concentrations (IU) of Cho, Cr, mI, lactate, and lipid, which all consistently have been reported to be abnormal in tumors, were analyzed. The unsuppressed water signal, assuming a water content of $75 \%$, was used as an internal concentration reference.

\section{Molecular Diagnostic Analysis}

SMARCB1 Protocol. SMARCB1 molecular genetic analysis by Sanger sequencing and multiplex ligation-dependent probe amplification were conducted on all tissue samples to confirm the diagnosis of $\mathrm{AT} / \mathrm{RT}^{11}$ per prior reported methodology.

ASCL1 Protocol/Immunohistochemistry and Automated Scoring of CKB. Immunohistochemical studies were performed as previously described. ${ }^{12}$ The primary antibody (ASCL1) (Mouse AntiMASH1, catalog No. 556604; BD Biosciences, San Jose, California) diluted 1:100 with Bond Primary Antibody Diluent (Leica Biosystems, Buffalo Grove, Illinois) was incubated for 30 minutes. The primary antibody was detected using the Bond Polymer Refine Detection kit (Leica Biosystems), which uses a peroxidase block, secondary antibodies, and 3, 3-diaminobenzidine and was finally counter-stained with hematoxylin.

Immunohistochemical studies for CKB were performed on previously characterized rhabdoid tumor tissue microarrays as 
previously described, ${ }^{13,14}$ and full paraffin-embedded sections from all available tumor blocks were obtained from imaged patients. Quantification of immunohistochemical data was performed as previously described. ${ }^{15}$ Blocker D, Streptavidin (HRP), and the DAB detection kit (Ventana Medical Systems, Tucson, Arizona) were used according to the manufacturer's instructions.

For automated scoring, each tissue microarray slide was scanned using a Scanscope Scanner (Aperio, Vista, California) and viewed through the ImageScope software program (Aperio). An individual blinded to the experimental design captured JPEG images from each core (circular area of $315 \mathrm{~cm}^{2}$ corresponding to the entire core) at $10 \times$ magnification. For full sections, the same blinded individual captured 5 random areas (circular area of $315 \mathrm{~cm}^{2}$ at $10 \times$ magnification). Quantification was conducted using an automated analysis program with the Matlab Image-Processing Toolbox (MathWorks, Natick, Massachusetts) based on previously described methodology. ${ }^{15}$ The final score for a given case and marker was calculated by averaging the score of 2 cores (for each case) or from 5 areas per section for full slides. These raw $\mathrm{CKB}$ numbers were log-transformed $\left(\mathrm{CKB}=\log _{10}\left[\mathrm{CKB}_{\text {raw }}\right]\right)$ for statistical analysis.

\section{Data Collection}

Frozen tumor tissue from 16 of 20 patients with AT/RTs and MR spectroscopy studies from all 20 patients diagnosed at Children's Hospital Los Angeles between 2000 and 2017 were available for analysis. Sufficient tissue samples available from 11 patients were examined for CKB.

\section{Statistical Analysis}

Statistical analysis was informed by the previously reported finding of 2 distinct molecular subtypes defined by the expression of the molecular marker ASCL1. ${ }^{10}$ For demographic measurements, the Fisher exact test was used to assess the difference in sex and lesion location (posterior fossa versus lesions outside the posterior fossa) between the 2 ATRT categories. A 2-sided exact test with a Wilcoxon score was used to examine the difference in continuous measurements, including the demographics, month of follow-up, and metabolic profiles. Because there were multiple outcomes, the sequential gatekeeper strategy ${ }^{16}$ was used to control the family-wise type I error rate. In this strategy, the outcomes were grouped into primary, secondary, and tertiary families. The primary and secondary outcomes were $\mathrm{CKB}$ and $\mathrm{Cr}$, respectively. The tertiary outcome family included Cho, lactate, $\mathrm{mI}$, and lipids. We first gave the full $\alpha$ of .05 to the primary outcome. If the primary outcome failed to reject the null hypothesis, the statistical test would stop at the primary level. Otherwise, the $\alpha$ value would be fully passed to the secondary outcome. If it failed to reject the null hypothesis at the secondary family, the statistical test would stop; otherwise, it would proceed to the tertiary outcome family. Because the tertiary family had 4 outcome measurements, the Benjamini-Hochberg false discovery rate correction method was used to control the error introduced by multiple testing. Furthermore, Spearman correlation analysis was used to assess the correlation between $\mathrm{CKB}$ and $\mathrm{Cr}$ concentrations in AT/RTs. We have conducted an exploratory analysis of survival using the KaplanMeier curve with a log-rank test. Patients who died from causes not related to disease progression were excluded because the log- rank test assumes noninformative right censoring. Patients at final follow-up were censored. SAS 9.4 (SAS Institute, Cary, North Carolina) was used for all statistical tests.

\section{RESULTS}

In vivo MR spectra acquired from AT/RTs demonstrated considerable metabolic heterogeneity. For example, whereas in some patients signals from $\mathrm{Cr}$ and $\mathrm{mI}$ were readily detectable, these metabolites were essentially absent in other AT/RT cases (Fig 1).

$\mathrm{CKB}$ was analyzed independently for $11 \mathrm{AT} / \mathrm{RT}$ s, and significantly higher CKB levels were observed in the cohort with ASCL1 expression $(P<.05$, Table 2$)$ in our patient population. The subsequent analysis of in vivo $\mathrm{Cr}$ concentrations demonstrated that Cr was significantly higher in tumors with ASCL1 expression than in tumors without ASCL1 expression $(P<.05$, Table 2$)$. In addition, significantly different in these 2 subgroups was the metabolite $\mathrm{mI}(P<.05$, Table 2$)$, whereas lipid levels approached significance $(P=.07$, Table 2$)$. Pearson correlation analysis showed statistically significant positive correlations of CKB with absolute $\mathrm{Cr}(P<.05)$ and $\mathrm{mI}(P<.01)$ concentrations.

AT/RTs located outside the posterior fossa more often expressed ASCL1 (6 versus 3 patients), whereas AT/RTs in the posterior fossa more often did not express ASCL1 (3 versus 4). Although this difference was not statistically significant in this small cohort of patients, the trend is consistent with the findings reported by Torchia et al. ${ }^{10}$ Overall survival in the 2 AT/RT subgroups was not significantly different $(P=.93)$.

\section{DISCUSSION}

AT/RTs are rare, aggressive central nervous system tumors that are predominantly encountered in very young children. These tumors have been considered incurable, with survival being typically $<1$ year after diagnosis for most patients. Although AT/RTs are uniformly defined by the loss of expression of SMARCB1, clinically there is considerable heterogeneity. While most children with these tumors have a very poor prognosis, a subset of patients experience distinctly prolonged survival, indicating epigenetic heterogeneity that potentially affects the response to treatment. ${ }^{6}$ Indeed, current consensus from a large multicenter study is that there are at least 3 molecular subgroups, referred to as AT/RTSHH, AT/RT-TYR, and AT/RT-MYC. ${ }^{8}$ A second and independent consortium of investigators ${ }^{7}$ also postulated 3 molecular subgroups of AT/RTs: Group 1 carried "neurogenic" signatures such as the expression of ASCL1 with clinically more favorable behavior, and groups 2A and 2B consisted of "mesenchymal" AT/ RTs, which were more aggressive/treatment-resistant and did not express ASCL1. Richardson et $\mathrm{al}^{5}$ suggested that AT/RT-SHH, AT/RT-TYR, and AT/RT-MYC subgroups may correspond with group 1, 2A, and 2B subgroups, respectively. However, this possibility has not been formally investigated and confirmed. In this study, to establish a clear separation of patients, we focused on the molecular feature of ASCL1 expression with our groups comprising AT/RTs that either expressed ASCL1 or did not express ASCL1.

When comparing in vivo MR spectroscopy measurements in AT/RTs, we observed significantly higher $\mathrm{Cr}$ concentrations in the ASCL1-expressing group versus the non-ASCL1-expressing group. $\mathrm{Cr}$ is an energy metabolite that is synthesized through a 

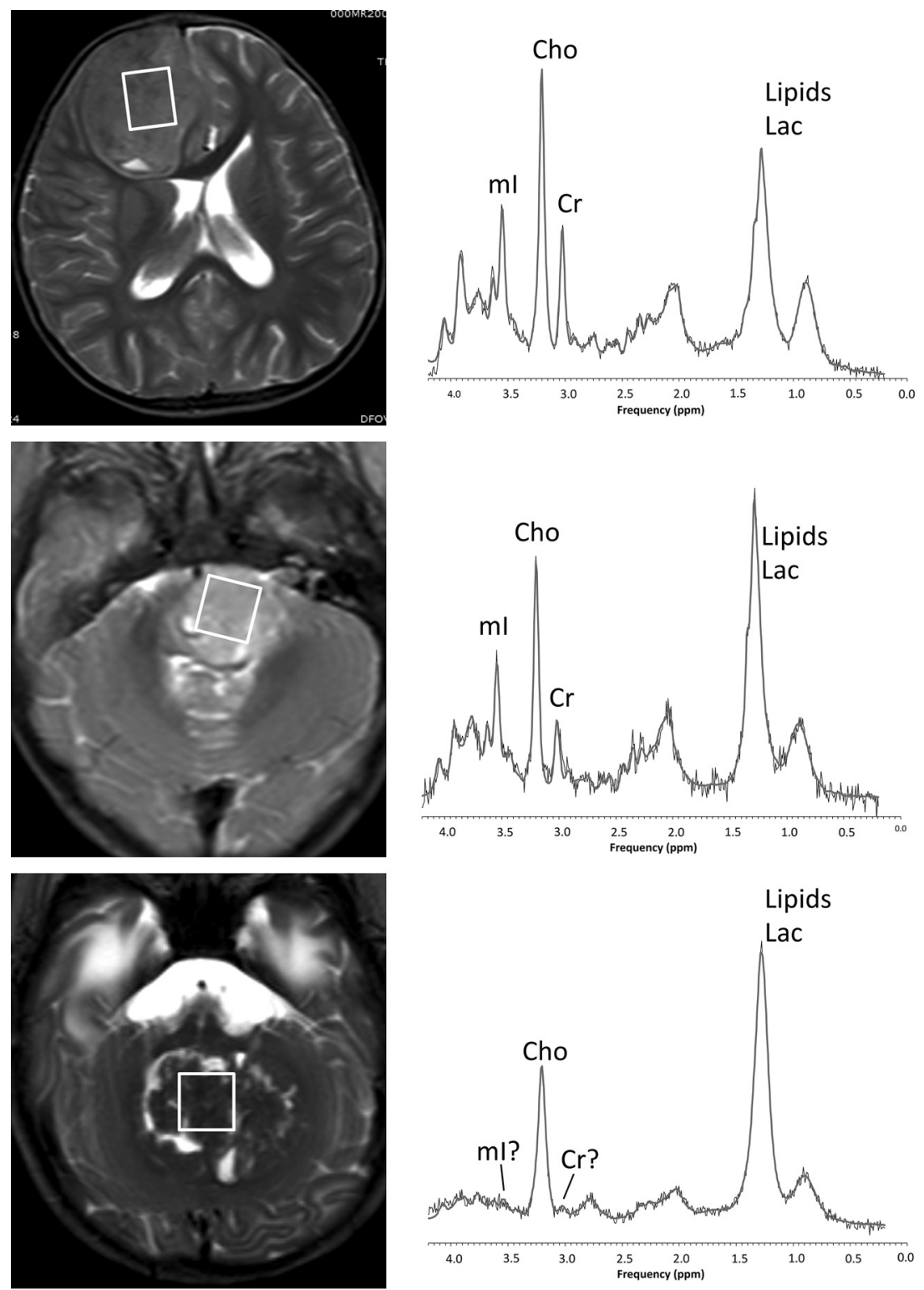

FIG 1. T2-weighted MR imaging and MR spectra of supratentorial AT/RTs with ASCL1 expression (upper row), a posterior fossa AT/RT with ASCL1 expression (middle row), and posterior fossa AT/RT without ASCLl expression (lower row). $\mathrm{Cr}$, Cho, and $\mathrm{ml}$ are readily detectable in the spectra of supratentorial and posterior fossa ASCL1-expressing AT/RTs, whereas only Cho is clearly detectable in the ASCL1-nonexpressing posterior fossa AT/RT. All spectra show prominent signal from lipids and lactate (Lac). Shown are the unprocessed data (thin black lines) with the superimposed fit (thick gray lines) used for quantification. The "box" seen in the images represents the voxel placement.

2-step process in the kidneys, pancreas, and liver. The brain then takes up Cr from the bloodstream by specific receptors known as SLC6A8, which are expressed in neurons and oligodendrocytes of the brain. Once Cr enters the CNS, it becomes part of the free creatine/phosphocreatine/creatine kinase system, which serves as an energy reservoir to maintain stable adenosine triphosphate levels. ${ }^{17,18}$ Both free $\mathrm{Cr}$ and creatine/phosphocreatine contribute to the total Cr peak, which is quantified by MR spectroscopy.

For our cohort, the finding of higher levels of CKB in ASCL1expressing AT/RTs provides additional evidence for considerable differences in Cr uptake and regulation in the ASCL1-expressing subgroup of AT/RTs. Furthermore, we have reviewed publicly accessible data from the studies of Torchia et $\mathrm{al}^{7,10}$ and have noted that in their dataset, both $\mathrm{CKB}$ and the creatine receptor (SLC6A8) were significantly higher in AT/RTs with ASCL1 expression compared with tumors without expression of ASCL1. Further studies are necessary to determine whether there is a direct relationship between ASCL1 expression and creatine metabolism. However, the above observations strongly indicate that the energy metabolism in the ASCL1-expressing subgroup could be different from that in the subgroup without ASCL1 expression. Therefore, we postulate that the molecular differences of the subgroups ultimately lead to differences in the underlying metabolism, which can be identified by noninvasive methods such as MR spectroscopy.

Myo-inositol was also significantly higher in ASCL1-expressing AT/RTs than in those without expression of ASCL1. Myo-inositol, a sugarlike molecule, is involved in signaling as part of the second messenger systems in the normal brain. ${ }^{19}$ Similar to creatine, $\mathrm{mI}$ is not synthesized inside brain cells but is derived from the diet or is synthesized in the kidneys. Myo-inositol is believed to be a putative marker of glial cells, particularly of astrocytes. ${ }^{20,21}$ It is thus generally high in astrocytomas but decreases when lesions transform to a higher grade. $^{22,23}$ Myo-inositol is involved in the activation of the protein kinase C, which leads to production of proteolytic enzymes that are found more often in malignant, aggressive primary cerebral tumors. ${ }^{22}$ For AT/RTs, mI may potentially serve as a surrogate predictor of more aggressive lesions - that is, lower $\mathrm{mI}$ predicts the more aggressive AT/RTs without expression of ASCL1. The cause for low $\mathrm{mI}$ in these tumors may be the lack of uptake and transport; however, this was not specifically investigated in this study, and further validation studies are needed.

In contrast to $\mathrm{Cr}$ and $\mathrm{mI}$, which were both lower in AT/RTs that did not demonstrate ASCL1 expression, lipids were more prominent, though statistical significance was not reached. There is some controversy about the origin of the lipid signal in tumors. Some groups suggested that the lipid signal originates from mobile lipid molecules as a result of tissue degradation and necrosis of aggressively growing lesions. ${ }^{24-26}$ On the other hand, prominent lipid signal was also reported in spectra obtained from tumors without detectable necrosis. ${ }^{27}$ In any case, there appears to 
Table 2: Features of AT/RTs with expression of ASCL1 ${ }^{a}$

\begin{tabular}{lccc}
\hline & $\begin{array}{c}\text { ASCL1 } \\
\text { Pos. }\end{array}$ & $\begin{array}{c}\text { ASCL1 } \\
\text { Neg. }\end{array}$ & $\begin{array}{c}P \\
\text { Value }\end{array}$ \\
\hline Age (mean) (yr) & $3.4 \pm 4.5$ & $1.8 \pm 0.8$ & NS \\
Sex (M/F) & $6: 3$ & $5: 2$ & NS \\
Location PF vs not PF & 3 vs 6 & 4 vs 3 & NS \\
Cr $^{\mathrm{b}}$ & $3.4 \pm 1.1$ & $1.8 \pm 0.8$ & $<.05$ \\
Cho $^{\mathrm{b}}$ & $3.2 \pm 0.8$ & $3.8 \pm 2.1$ & $\mathrm{NS}$ \\
ml $^{\mathrm{b}}$ & $9.0 \pm 1.5$ & $4.7 \pm 3.6$ & $<.05$ \\
Lac $^{\mathrm{b}}$ & $5.0 \pm 3.9$ & $2.6 \pm 2.6$ & $\mathrm{NS}$ \\
Lipids $^{\mathrm{b}}$ & $44 \pm 20$ & $80 \pm 30$ & $\mathrm{NS}$ \\
CKB (mean) & $6.3 \pm 0.3$ & $5.4 \pm 0.5$ & $<0.05$ \\
Months of follow-up (mean) & $22 \pm 14$ & $32 \pm 27$ & $\mathrm{NS}$ \\
\hline
\end{tabular}

Note:-NS indicates not significant; Pos., positive; Neg., negative; PF, posterior fossa.

${ }^{a}$ Tissue from 16 of 20 AT/RTs was available for testing of ASCL1 status.

${ }^{\mathrm{b}}$ Means in IU.

${ }^{c}$ Approaching significance with $P=.07$.

be consensus that the presence of high lipids is generally an indicator of more malignant lesions. ${ }^{24}$ The observed trend of higher lipid levels in AT/RTs without expression of ASCL1 would thus be consistent with a potentially worse prognosis.

Differences in our patient cohort in terms of germline-versussporadic SMARCB1 mutations were also investigated. A total of 4 patients were identified with germline mutations (Table 1), all of which demonstrated expression of ASCL1. Although this is an interesting finding, more data are needed to investigate the clinical significance, if any, of this observation.

Overall survival in the $2 \mathrm{AT} / \mathrm{RT}$ subgroups in this study was not significantly different. This appears to be inconsistent with the study of Torchia et al, ${ }^{10}$ which predicted a clinically more favorable outcome for AT/RTs with expression of ASCL1. However, treatment approaches for most patients included in this study were not consistent, likely playing a role in outcome/prognosis. Thus, the inability of this study to detect a difference in clinical outcomes of a small group of AT/RTs should not be overemphasized. Our study did, however, demonstrate a trend concordant with the findings of Torchia et $\mathrm{al}^{7}{ }^{7}$ specifically that AT/RTs expressing ASCL1 are more often located outside the posterior fossa.

The question arises as to whether metabolic features of AT/ RTs, obtained by noninvasive MR spectroscopy, form clusters and whether these clusters correlate with molecular subtypes or specific molecular features. Our analysis of the MR spectroscopy data available for this study is suggestive of the presence of 2 dominant metabolic clusters consisting of AT/RTs with significant differences in $\mathrm{Cr}$ and $\mathrm{mI}$ (relative to Cho) (Fig 2). However, given the broad heterogeneity of the larger metabolic group data points (dashed line circle in Fig 2), it is unclear whether these cases form a separate third group as predicted by the molecular phenotypes. Further, multicenter prospective trials with larger number of subjects using MR spectroscopy data are needed for analysis.

Our study had several limitations, including the single-center retrospective design. Our total number of patients is small. However, to our knowledge, this is the largest cohort of patients with AT/RTs with both molecular and metabolic (MR spectroscopy) data. No corrections of metabolite concentrations for T1 and T2 effects were applied because they are unknown in AT/RTs and it was not feasible to measure them in a clinical setting in patients. It is known that $\mathrm{T} 1$ times are longer at $3 \mathrm{~T}$ than at $1.5 \mathrm{~T}$. The longer

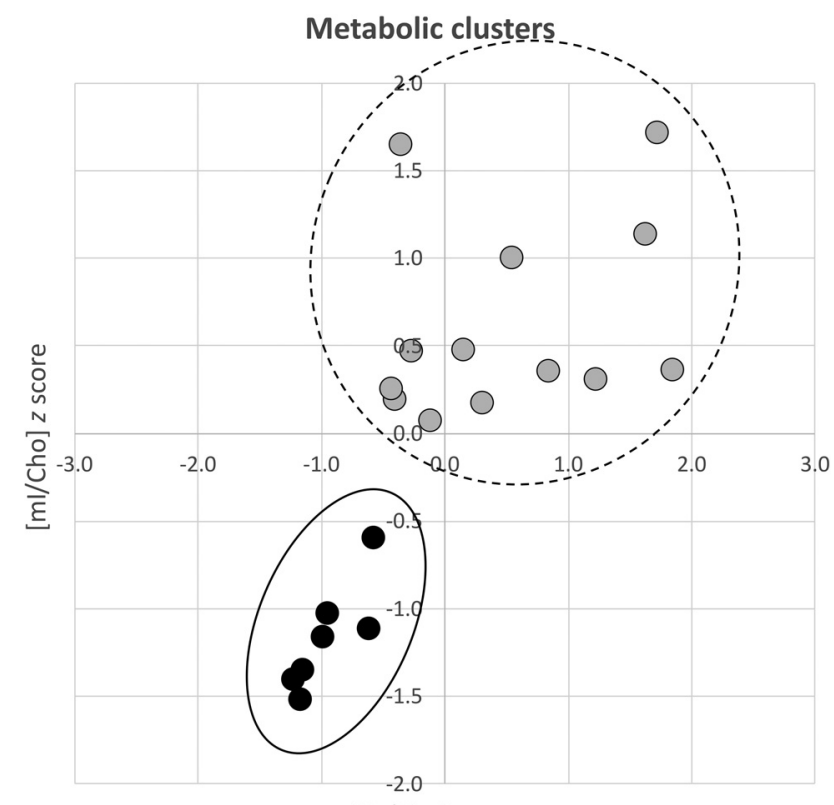

[Cr/Cho] z score

FIG 2. K-means cluster analysis graph demonstrating the metabolic clusters based on $\mathrm{Cr} / \mathrm{Cho}$ and $\mathrm{ml} / \mathrm{Cho}$ ratios. Ratios were transformed to $z$ scores for better illustration. Findings suggest that a subgroup of AT/RTs (solid line) are homogeneously characterized by very low $\mathrm{Cr}$ and $\mathrm{ml}$ levels (relative to $\mathrm{Cho}$ ) and are well-separated from other AT/RTs. It is, however, unclear whether the remaining larger cluster of AT/RTs (dashed line) forms a single group or multiple separate groups as suggested by the molecular data.

TR of our 3T studies (2.0 versus 1.5 seconds) partially corrects for this effect. Additionally, due to acquisition of the data at both 1.5 and 3T, differentiation of the $\mathrm{mI}$ and glycine peak proved to be challenging, particularly at $1.5 \mathrm{~T}$ because both $\mathrm{mI}$ and glycine contribute to the peak at $3.5 \mathrm{ppm}$. Clinically, glycine and $\mathrm{mI}$ may have similar implications because prior studies have reported that glycine consumption and synthesis are correlated with rapid cancer cell proliferation ${ }^{28}$; therefore, glycine may serve as a marker for more aggressive tumors. Changes in glycine therefore can potentially coincide with and/or parallel those of $\mathrm{mI}$ in aggressive brain tumors. Further studies at $3 \mathrm{~T}$ are warranted to investigate the role of glycine in aggressive pediatric brain tumors.

\section{CONCLUSIONS}

Our study demonstrates that metabolic profiles as determined by MR spectroscopy can help predict molecular features of AT/RTs, potentially serving as in vivo biomarkers for the molecular subgroups. AT/RTs with and without expression of ASCL1 are currently treated using similar aggressive chemotherapy regimens, sometimes with added radiation therapy. However, it is expected that in the future, therapeutic options will be developed that will try to exploit unique biologic/molecular features in individual patients. A noninvasive method for early stratification would then be helpful clinically and is the ultimate goal for the current precision-based oncologic management of patients with brain tumors.

Disclosures: Benita Tamrazi—RELATED: Grant: American Society of Pediatric Neuroradiology Research Grant, Comments: The grant supports research conducted for this article*; UNRELATED: Consultancy: Bayer. Debra Hawes—UNRELATED: Employment: University of Southern California, Comments: primary employer Children's Hospital Los Angeles. Marvin Nelson—UNRELATED: Employment: Children's Hospi- 
tal Los Angeles Medical Group; Comments: book published in 2012. Stefan BlümlUNRELATED: lans Friends Foundations, Rudi Schulte Research Institute, Comments: Salary support. *Money paid to the institution.

\section{REFERENCES}

1. Ostrom QT, Gittleman H, Fulop J, et al. CBTRUS Statistical Report: Primary Brain and Central Nervous System Tumors Diagnosed in the United States in 2008-2012. Neuro Oncol 2015;17(Suppl 4): iv1-62 CrossRef Medline

2. Gurney J, Smith M, Bunin G. CNS and miscellaneous intracranial and intraspinal neoplasms. In: Ries L, Smith M, Gurney J, et al., eds. Cancer Incidence and Survival among Children and Adolescents: United States SEER Program 1975-1995. Bethesda: National Cancer Institute, SEER Program. NIH Pub. No. 99-4649; 1999

3. Biegel JA, Zhou JY, Rorke LB, et al. Germ-line and acquired mutations of INI1 in atypical teratoid and rhabdoid tumors. Cancer Res 1999;59:74-79 Medline

4. Versteege I, Sévenet N, Lange J, et al. Truncating mutations of hSNF5/INI1 in aggressive paediatric cancer. Nature 1998;394: 203-06 CrossRef Medline

5. Richardson EA, Ho B, Huang A. Atypical teratoid rhabdoid tumour: from tumours to therapies. J Korean Neurosurg Soc 2018;61:302-11 CrossRef Medline

6. Ginn KF, Gajjar A. Atypical teratoid rhabdoid tumor: current therapy and future directions. Front Oncol 2012;2:114 CrossRef Medline

7. Torchia J, Golbourn B, Feng S, et al. Integrated (epi)-genomic analyses identify subgroup-specific therapeutic targets in CNS rhabdoid tumors. Cancer Cell 2016;30:891-908 CrossRef Medline

8. Johann PD, Erkek S, Zapatka M, et al. Atypical teratoid/rhabdoid tumors are comprised of three epigenetic subgroups with distinct enhancer landscapes. Cancer Cell 2016;29:379-93 CrossRef Medline

9. Nowak J, Nemes K, Hohm A, et al. Magnetic resonance imaging surrogates of molecular subgroups in atypical teratoid/rhabdoid tumor. Neuro Oncol 2018;20:1672-79 CrossRef Medline

10. Torchia J, Picard D, Lafay-Cousin L, et al. Molecular subgroups of atypical teratoid rhabdoid tumours in children: an integrated genomic and clinicopathological analysis. Lancet Oncol 2015;16: 569-82 CrossRef Medline

11. Eaton KW, Tooke LS, Wainwright LM, et al. Spectrum of SMARCB1/ INI1 mutations in familial and sporadic rhabdoid tumors. Pediatr Blood Cancer 2011;56:7-15 CrossRef Medline

12. Panwalkar P, Clark J, Ramaswamy V, et al. Immunohistochemical analysis of $\mathrm{H} 3 \mathrm{~K} 27 \mathrm{me} 3$ demonstrates global reduction in group-A childhood posterior fossa ependymoma and is a powerful predictor of outcome. Acta Neuropathol 2017;134:705-14 CrossRef Medline

13. Venneti S, Le P, Martinez D, et al. Malignant rhabdoid tumors express stem cell factors, which relate to the expression of EZH2 and Id proteins. Am J Surg Pathol 2011;35:1463-72 CrossRef Medline
14. Eaton BT, Hyatt AD, Brookes SM. The replication of bluetongue virus. Curr Top Microbiol Immunol 1990;162:89-118 Medline

15. Venneti S, Garimella MT, Sullivan LM, et al. Evaluation of histone 3 lysine 27 trimethylation $(\mathrm{H} 3 \mathrm{~K} 27 \mathrm{me} 3)$ and enhancer of Zest 2 $(\mathrm{EZH} 2)$ in pediatric glial and glioneuronal tumors shows decreased H3K27me3 in H3F3A K27M mutant glioblastomas. Brain Pathol 2013;23:558-64 CrossRef Medline

16. FDA. Multiple Endpoints in Clinical Trials. 2017. https://www.fda. gov/downloads/drugs/guidancecomplianceregulatoryinformation/ guidances/ucm536750.pdf. Accessed September 18, 2018

17. Hanna-El-Daher L, Braissant O. Creatine synthesis and exchanges between brain cells: what can be learned from human creatine deficiencies and various experimental models? Amino Acids 2016;48: 1877-95 CrossRef Medline

18. Braissant O, Henry H, Villard AM, et al. Creatine synthesis and transport during rat embryogenesis: spatiotemporal expression of AGAT, GAMT and CT1. BMC Dev Biol 2005;5:9 CrossRef Medline

19. Fisher SK, Novak JE, Agranoff BW. Inositol and higher inositol phosphates in neural tissues: homeostasis, metabolism and functional significance. J Neurochem 2002;82:736-54 CrossRef Medline

20. Isaacks RE, Bender AS, Kim CY, et al. Osmotic regulation of myoinositol uptake in primary astrocyte cultures. Neurochem Res 1994; 19:331-38 CrossRef Medline

21. Brand A, Richter-Landsberg C, Leibfritz D. Multinuclear NMR studies on the energy metabolism of glial and neuronal cells. $\mathrm{Dev} \mathrm{Neu}$ rosci 1993;15:289-98 CrossRef Medline

22. Castillo M, Smith JK, Kwock L. Correlation of myo-inositol levels and grading of cerebral astrocytomas. AJNR Am J Neuroradiol 2000; 21:1645-49 Medline

23. Panigrahy A, Krieger MD, Gonzalez-Gomez I, et al. Quantitative short echo time 1H-MR spectroscopy of untreated pediatric brain tumors: preoperative diagnosis and characterization. AJNR Am J Neuroradiol 2006;27:560-72 Medline

24. Negendank W, Sauter R. Intratumoral lipids in $\mathbf{1 H}$ MRS in vivo in brain tumors: experience of the Siemens cooperative clinical trial. Anticancer Res 1996;16:1533-38 Medline

25. Kuesel AC, Sutherland GR, Halliday W, et al. 1H MRS of high grade astrocytomas: mobile lipid accumulation in necrotic tissue. NMR Biomed 1994;7:149-55 CrossRef Medline

26. Rock JP, Hearshen D, Scarpace L, et al. Correlations between magnetic resonance spectroscopy and image-guided histopathology, with special attention to radiation necrosis. Neurosurgery 2002;51: 912-19, discussion 919-20 Medline

27. Kuesel AC, Donnelly SM, Halliday W, et al. Mobile lipids and metabolic heterogeneity of brain tumours as detectable by ex vivo $1 \mathrm{H}$ MR spectroscopy. NMR Biomed 1994;7:172-80 CrossRef Medline

28. Jain M, Nilsson R, Sharma S, et al. Metabolite profiling identifies a key role for glycine in rapid cancer cell proliferation. Science 2012; 336:1040-44 CrossRef Medline 